\title{
How Digital Transformation Shapes Corporate IT: Ten Theses about the IT Organization of the Future
}

\author{
Frederik Ahlemann \\ University of Duisburg-Essen Essen, Germany \\ Email: frederik.ahlemann@uni-due.de
}

D IGITAL transformation is a major challenge for many organizations. IT managers in particular not only wonder what the next digital trends in their industry will be, they also need to understand how today's IT organizations will change in light of digital transformation. I will first discuss some foundations of digital transformation and will then present 10 theses on how digital transformation will influence corporate IT.

Thesis 1: There will be no business without IT: IT is the indispensable driver and enabler of value creation

IT is already the backbone of many enterprises and a key resource. At the same time, many executives still do not view it as a crucial competitive factor. Digital transformation will change this. IT will not only be used to automate internal and external business processes. It will also be used to realize new digital products, services, and business models. Beyond this, IT will fundamentally change the ways enterprises will be organized and managed. Even many demanding management tasks can be performed by AI systems fueled by machine learning. This will revolutionize how enterprises operatein terms of speed, reliability, efficiency, and quality. At the same time, business will increase its dependency on IT. System crashes that cannot be fixed immediately will lead to insolvency faster than ever before owing to interrupted business operations.

Thesis 2: Development and operations will lose weight: Tomorrow's IT functions will follow the paradigm of Innovatedesign-transform

Innovate-design-transform Classic corporate IT follows the Plan-build-run paradigm, which serves as a blueprint for the structure of an IT organization and places significant emphasis on long-term planning and subsequent implementation in a more or less stable environment. The focus is on efficiency. However, digital transformation requires enterprises to become more flexible so as to realize innovative business models. Thus, future IT functions will follow the Innovate-design-transform paradigm that stress the importance of innovation, subsequent design of IS with high acceptance and adoption rates, and a transformation of the organization to serve the new business model.
Thesis 3: Shadow IT becomes normal: IT innovations are developed through joint interdisciplinary teams in the business departments

Today, in most enterprises, IT projects are initiated by the business and then realized in the IT organization. However, this process neglects some key characteristics of innovation in the digital age: (1) innovations must be developed quickly, (2) innovations are the result of a (very) close collaboration of business and IT (and external parties), (3) requirements change rapidly, (4) communication is intense and frequent. Thus, in the future, IT professionals will be part of the business department to continuously work on digital innovations, whether these be process, product, or business model innovations. At the same time, the business can decide, with some constraints, on the IT/IS they need.

Thesis 4: Innovations through networks: Strategic vendors become innovation partners Most of today's IT organizations struggle to implement

Most of today's IT organizations struggle to implement disruptive IT innovations because they lack the required capabilities. For instance, many businesses have ideas regarding the use of big data and machine learning. However, they lack the data scientists necessary to implement these concepts. Thus, digital partnerships and digital innovation networks will become more important. These partnerships and networks will often be very different from classic vendor relationships. They are long-term, eye-level, strategic, and will also often involve benefits-sharing.

Thesis 5: From applications to user: Development processes are agile, user-centric, and closely linked to IT operations

Even today, software development is often based on the waterfall model. The timespan between an initial idea and handover to IT operations is fairly long. In times of digital innovation, this can significantly hinder the gaining of market shares quickly and the building of positive brand images. Future IT will be lightweight, characterized by agile development processes, and free from too many architectural and organizational constraints. It will allow developers to focus on user needs and user feedback. High acceptance rates and intense usage are primary goals of development. Developers will iteratively improve applications to satisfy users, and mostly in very short cycles. A close integration of development and IT operations is key for this approach (DevOps). 
Thesis 6: Infrastructure as a commodity: IT infrastructure services will be traded on free markets

Despite the trend towards outsourcing, many companies still operate their own IT infrastructure in data centers. The decision to have an own IT infrastructure is based on a few assumptions. For instance, organizations believe that an own IT infrastructure allows for better controlling, higher security levels, better compliance, and a better cost structure. These assumptions will soon be invalid, if they are not already invalid. In the future, data centers will no longer be necessary, and corporate IT will completely be based on public cloud offerings, with very few exceptions. These public cloud offerings will largely be standardized, allowing for trading at new types of exchanges for IT services.

Thesis 7: Digital transformation as a major risk: Security and business continuity will be primary cross- departmental functions The growing use of IT-even as parts of products and services

The growing use of IT-even as parts of products and services-will increase dependency and vulnerability. Attacks against central IT will directly jeopardize a company's continued existence. Thus, security and business continuity management (SBCM) will gain importance. It will pervade all areas of an enterprise and will no longer be a field of only IT experts. Businesses will realize that SBCM is an indispensable tool for long-term business success, because attacks and threats from the outside as well as from the inside will become 'normal'.

Thesis 8: Transformable IT landscapes: IT architectures will be standardized, modular, flexible, ubiquitous, elastic, costefficient, and secure

Today's complex IT landscapes will undergo tremendous transformation. Via practices such as enterprise architecture management (EAM), increased standardization both at the industry and company levels, technological advances and the trends towards cloud computing, the IT architectures of the future will leave many current challenges behind. We expect them to be more standardized (through cloud computing and industry starndardization), more modular (through technological and architectural advances), more flexible (through tech- nological advances), ubiquitous (through mobile computing, new device categories, more flexible architectures), elastic (through cloud computing), more cost- efficient (through cloud computing), and more secure (through technological advances and cloud providers' expertise). This will allow for more dynamic, fast, and easy implementation of new products, services, and business models.

Thesis 9: The end of the IT department: IT experts will be part of business departments

Given that the aforementioned trends come on-stream, the largest share of IT specialists will likely work on the specification, development, configuration/customization, and maintenance of applications. However, because close collaboration with business is key for the success of these activities, we expect them to become part of the business departments, where they can sit next to users and business managers and can develop new products, services, and processes necessary to make IT innovations happen. The IT department will shrink significantly, because fewer infrastructure experts are required (owing to the use of cloud services) and the move of IS specialists to the business. The remaining unit will focus on more strategic tasks such as EAM, SBCM, procurement, partner management, innovation management, and portfolio management. Since these functions are crucial for a corporate success, we expect them to be located alongside the board.

Thesis 10: Demographics, digital natives, and individual entrepreneurship: Employees become a strategic competitive factor

A key factor for the success of digital initiatives today and in the future will be access to skilled human resources. Digital transformation requires specific qualifications and skills that are currently fairly rare. Even in the future, with new study programs at universities (e.g. for data science), it is likely that the professionals required will be scarce. The demographic changes in many western societies, the changes in young professionals' value systems, and the growing desire for individualism and self-determination will make a dedicated and innovative IT human resource management necessary. 